\section{Tasa de recidivismo en urgencias por dorsalgia y lumbalgia en función del tratamiento administrado tras una primera valoración}

\section{Resumen}

El recidivismo en urgencias es la demanda asistencial generada por pacientes que ya han sido valorados previamente por el mismo motivo o por un motivo derivado por el tratamiento indicado. El propósito del presente estudio es determinar en qué medida influye el tipo de tratamiento analgésico pautado en los procesos dolorosos axiales dorsales y lumbalgia en el recidivismo. Se realizó un estudio retrospectivo sobre 24490 registros de pacientes que consultaron por estos motivos en urgencias entre 2010 y 2015. Se identificaron la existencia de consultas posteriores hasta cumplidos 30 días y el tipo de tratamiento analgésico pautado. En general el $18.02 \%$ de los pacientes consultaron nuevamente antes del primer mes. A los 30 días la proporción de pacientes que no habían reconsultado fue $67.4 \%$, 78.6\% y $91.7 \%$, según fueron tratados con medicamentos del primer, segundo o tercer escalón analgésico. En todos los periodos de observación, el mal control analgésico fue el principal motivo de reconsulta. La identificación de eventos adversos como causa de consulta repetida se presentó mayoritariamente en pacientes que recibieron fármacos del segundo escalón analgésico.

Aunque se requiere estudios prospectivos y controlados para validar el valor protector de ciertas prescripciones contra el recidivismo en urgencias, nuestros resultados sugieren intensamente que el uso de fármacos del tercer escalón puede tener un impacto positivo en este indicador.
Carlos Guillén Astete ${ }^{1,2}$, Miguel Zamorano

Serrano², César Carballo Carmona ${ }^{3}$, Roberto Penedo Alonso $^{2}$

1 Servicio de Reumatología, Hospital Universitario Ramón y Cajal. Madrid. España

2 Servicio de Urgencias, Hospital Universitario Ramón y Cajal. Madrid. España

3 Servicio de Urgencias, Hospital Universitario La Paz. Madrid. España

Correspondencia:

Carlos Antonio Guillén Astete

” cguillen.hrc@salud.madrid.org 


\section{Abstract}

Urgency recidivism is defined as further need of medical assessment after a initial consultation due to the same chief complain or to an adverse effect caused by the original prescription. Aim of this study is to determine in what extent, the kind of analgesic treatment influences recidivism in patients who consulted by axial pain. A retrospective study based on 24490 records from 2010 to 2015 was performed. A survival curve was performed for each group of treatments. Globally, $18.02 \%$ of patients consulted before the first month. At the thirtieth day the proportion of patients who did not consulted again was $67.4 \%, 78.6 \%$ and $91.7 \%$, according to have been treated with medications of the first, second or third analgesic step, respectively. In all periods of follow up, pain control failure was the most frequent chief complain, however, adverse effects of prescription were identified as major cause of consultation among patients who were treated with medications of the second analgesic step. Further prospective controlled studied should be performed to validate the protector value of certain prescriptions against urgency recidivism, however, our results strongly suggest that the use of medications of the third analgesic step could have a positive effect in this measure.

\section{Introducción}

La patología del aparato locomotor y especialmente la musculoesquelética concentra una importante proporción de la consultas en los servicios de urgencias[1-3].

El recidivismo, entendido como la demanda de atención médica repetida de un paciente por el mismo motivo o por motivos derivados de un acto médico previo, contribuyen a incrementar la carga asistencial[4,5].

El uso de fármacos opiáceos son una alternativa reconocida al manejo del dolor musculoesquelético de tipo disruptivo sin embargo se considera que debido a sus potenciales efectos adversos su uso en ancianos se ve limitado[6-9].

En nuestra experiencia reciente, el uso de opiáceos mayores no ha representado un incremento significativo de eventos adversos respecto de otras terapias sin embargo, ha demostrado un buen control sintomático con consecuente reducción en el número de visitas repetidas[10,11].

El objetivo de este estudio es determinar la tasa de supervivencia de pacientes que han consultado por urgencias por procesos dolorosos relacionados con el esqueleto axial en función del tipo de tratamiento pautado en la primera consulta. 


\section{Material y método}

Se realizó un estudio de tipo descriptivo transversal sobre historias clínicas de pacientes que consultaron por nuestro servicio de urgencias entre Enero de 2010 y Diciembre de 2015.

Los registros incluidos correspondieron a pacientes cuyo motivo de consulta fue dorsalgia o lumbalgia.

La búsqueda de registros se realizó mediante el programa informático Excalibur $®$, atendiendo al campo de motivo de consulta y diagnóstico final. En el campo de motivo de consulta de utilizaron los términos genéricos dorsalgia y lumbalgia y los términos adicionales "dolor lumbar", "lumbago", "ciática", "lumbocíatica", "dolor dorsal", "dolor de espalda", "dolor axial" y "raquialgia". Se excluyeron las algias de territorio cervical. En el campo de diagnóstico se utilizaron los términos de búsqueda "lumbago", "lumbalgia", "aplastamiento vertebral", "acuñamiento vertebral", "dorsalgia", "raquialgia", "ciática" y "lumbociática".

Una vez identificados los registros se incluyeron solamente aquellos en los que los datos demográficos de edad y sexo, y los datos referentes al tratamiento pautado al alta estuviesen disponibles.

Se utilizó el programa informático Horus (Servicio Madrileño de Salud, Consejería de Salud®) para acceder a los registros de valoraciones médicas posteriores al alta en los primeros 30 días.

Para efectos del estudio de supervivencia se agruparon a los pacientes según el tratamiento pautado al alta. Grupos se establecieron según la clasificación de la escala analgésica de la Organización Mundial de la Salud[12]. Un paciente se consideró de un determinado grupo según el fármaco de mayor escalón prescrito. Se consideró evento a: necesidad de consulta médica por el mismo motivo de consulta inicial, por mal control analgésico, por ingreso hospitalario, por efecto adverso de la medicación administrada -según criterio médico expresamente reseñado en el registro correspondiente-, y por necesidad de modificación del tratamiento pautado.

Aquellos pacientes que fueron ingresados el día de la primera valoración fueron excluidos del estudio.

No se discriminaron los registros según el mecanismo de la lesión.

El presente estudio contó con la aprobación del Comité de Ética e Investigación Científica de nuestro centro.

\section{Resultados}

Se identificaron 24530 registros a través de la búsqueda por campos. De ellos, se incluyeron finalmente 24490. Del total de registros incluidos, 19647 (80.2\%) correspondieron a lumbalgias y $4843(19.7 \%)$ a dorsalgias. Catorcemil doscientos cuatro registros correspondieron a pacientes del sexo femenino (57.9\%).

La cifra absoluta de lumbalgias y dorsalgias año a lo largo de los 5 periodos anuales estudiados así como su distribución porcentual según estratos de edad se detalla en la tabla 1.

La distribución porcentual global de lumbalgias en función del grupo de edad fue 5.8, 7.7, 10, 14.1, $21.7,20.6,18.2$ y $1.9 \%$ para los grupos $16-30,30-$ 40, 40-50, 50-60, 60-70, 70-80, 80-90 y más de 90 años, respectivamente. La distribución porcentual global de dorsalgias en función del grupo de edad fue 3.0, 7.0, 7.7, 15.3, 31.9, 19.3, 12.6 y 3.1\% para los mismos grupos.

Entre quienes consultaron por lumbalgia, fueron tratados con fármacos del primer escalón analgésico 4524 pacientes (18.4\%) mientras que 12888 (52.6\%) fueron tratados con al menos un fármaco del segundo escalón analgésico y 2253 (9.1\%) con al menos un fármaco del tercer escalón analgésico. Por otra parte, entre quienes consultaron por dorsalgia, fueron tratados con fármacos del primer escalón analgésico 1836 pacientes (37.9\%), mientras que 2548 (52.6\%) fueron tratados con al menos 
Tabla 1. Distribución de los motivos de consulta y/o diagnósticos finales en función del grupo de edad y año de extracción de registros.

\begin{tabular}{|c|c|c|c|c|c|c|c|c|c|c|}
\hline \multirow{2}{*}{$\begin{array}{c}\text { Grupo } \\
\text { edad }\end{array}$} & \multicolumn{7}{|c|}{ Año de extracción de registros de pacientes con lumbalgia } \\
\hline & $\mathbf{2 0 1 0 - 2 0 1 1}$ & $\%$ & $\mathbf{2 0 1 1 - 2 0 1 2}$ & $\%$ & $\mathbf{2 0 1 2 - 2 0 1 3}$ & $\%$ & $\mathbf{2 0 1 3 - 2 0 1 4}$ & $\%$ & $\mathbf{2 0 1 4 - 2 0 1 5}$ & $\%$ \\
\hline $16-30$ & 299 & $7,5 \%$ & 280 & $7,2 \%$ & 270 & $6,9 \%$ & 255 & $6,5 \%$ & 231 & $5,8 \%$ \\
\hline $30-40$ & 350 & $8,8 \%$ & 341 & $8,7 \%$ & 325 & $8,3 \%$ & 330 & $8,5 \%$ & 305 & $7,7 \%$ \\
\hline $40-50$ & 465 & $11,7 \%$ & 450 & $11,5 \%$ & 442 & $11,2 \%$ & 400 & $10,3 \%$ & 395 & $10,0 \%$ \\
\hline $50-60$ & 602 & $15,2 \%$ & 590 & $15,1 \%$ & 588 & $15,0 \%$ & 542 & $13,9 \%$ & 560 & $14,1 \%$ \\
\hline $60-70$ & 812 & $20,5 \%$ & 802 & $20,6 \%$ & 820 & $20,9 \%$ & 860 & $22,1 \%$ & 858 & $21,7 \%$ \\
\hline $70-80$ & 752 & $19,0 \%$ & 780 & $20,0 \%$ & 802 & $20,4 \%$ & 810 & $20,8 \%$ & 815 & $20,6 \%$ \\
\hline $80-90$ & 630 & $15,9 \%$ & 610 & $15,6 \%$ & 622 & $15,8 \%$ & 631 & $16,2 \%$ & 720 & $18,2 \%$ \\
\hline $90+$ & 53 & $1,3 \%$ & 48 & $1,2 \%$ & 60 & $1,5 \%$ & 67 & $1,7 \%$ & 75 & $1,9 \%$ \\
\hline
\end{tabular}

\begin{tabular}{|c|c|c|c|c|c|c|c|c|c|c|}
\hline $\begin{array}{c}\text { Grupo } \\
\text { edad }\end{array}$ & \multicolumn{7}{|c|}{ Año de extracción de registros de pacientes con dorsalgia } \\
\hline & $\mathbf{2 0 1 0 - 2 0 1 1}$ & $\%$ & $\mathbf{2 0 1 1 - 2 0 1 2}$ & $\%$ & $\mathbf{2 0 1 2 - 2 0 1 3}$ & $\%$ & $\mathbf{2 0 1 3 - 2 0 1 4}$ & $\%$ & $\mathbf{2 0 1 4 - 2 0 1 5}$ & $\%$ \\
\hline $16-30$ & 33 & $3,5 \%$ & 29 & $3,0 \%$ & 25 & $2,6 \%$ & 26 & $2,7 \%$ & 32 & $3,2 \%$ \\
\hline $30-40$ & 82 & $8,7 \%$ & 78 & $8,1 \%$ & 65 & $6,7 \%$ & 64 & $6,6 \%$ & 60 & $6,0 \%$ \\
\hline $40-50$ & 85 & $9,0 \%$ & 80 & $8,3 \%$ & 78 & $8,1 \%$ & 79 & $8,1 \%$ & 64 & $6,4 \%$ \\
\hline $50-60$ & 161 & $17,1 \%$ & 155 & $16,0 \%$ & 150 & $15,6 \%$ & 147 & $15,1 \%$ & 140 & $14,0 \%$ \\
\hline $60-70$ & 290 & $30,8 \%$ & 296 & $30,6 \%$ & 301 & $31,2 \%$ & 306 & $31,5 \%$ & 336 & $33,7 \%$ \\
\hline $70-80$ & 160 & $17,0 \%$ & 180 & $18,6 \%$ & 188 & $19,5 \%$ & 190 & $19,5 \%$ & 205 & $20,6 \%$ \\
\hline $80-90$ & 105 & $11,1 \%$ & 120 & $12,4 \%$ & 125 & $13,0 \%$ & 126 & $13,0 \%$ & 130 & $13,0 \%$ \\
\hline $90+$ & 26 & $2,8 \%$ & 30 & $3,1 \%$ & 32 & $3,3 \%$ & 34 & $3,5 \%$ & 30 & $3,0 \%$ \\
\hline
\end{tabular}

un fármaco del segundo escalón analgésico y 459 (9.5\%) con al menos un fármaco del tercer escalón analgésico. La distribución de tratamientos entre grandes grupos de edad se detalla en la tabla 2 .

Incluyendo a todos los pacientes independiente de su motivo de consulta o diagnóstico, en el grupo de 16-40 años, la prescripción de fármacos del primer escalón analgésico fue significativamente mayor que el resto de pautas $(28.9 \%, 9.5 \%$ y $6.4 \%$, respectivamente. $P<0.001)$. En el grupo de 70 o más años, la prescripción de fármacos del primer escalón analgésico fue significativamente menor que el resto de pautas $(20.9 \%, 43.7 \%$ y $39.9 \%$, respectivamente. $P<0.001)$. El Gráfico 1 resume la distribución de tratamientos en función de los grupos mayores de edad.

La tasa de recidivismo global (lumbalgia y dorsalgia) en los primeros 30 días de la consulta inicial fue $18.02 \%$. Entre los pacientes con lumbalgia como motivo de consulta o diagnóstico, a los 7 días de haberse realizado la valoración en urgencias, $93.2 \%$ de los tratados con el primer escalón analgésico, $92.4 \%$ de los que fueron tratados con el segundo escalón analgésico y $96.7 \%$ de los que 
Tabla 2. Distribución de los tipos de tratamiento entre grupos de edad.

\begin{tabular}{|l|c|c|c|c|c|c|c|c|}
\hline \multirow{2}{*}{ Grupo analgésico OMS } & \multicolumn{7}{|c|}{ Grupo de edad (años) - Lumbalgia } & \\
\cline { 2 - 11 } & $\mathbf{1 6 - 4 0}$ & $\%$ & $\mathbf{4 0 - 7 0}$ & $\%$ & $\mathbf{7 0 - 9 0 +}$ & $\%$ & Total & $\%$ \\
\hline Primer escalón & 1550 & $51,9 \%$ & 2130 & $23,2 \%$ & 844 & $11,3 \%$ & 4524 & $18,4 \%$ \\
\hline Segundo escalón & 1267 & $42,4 \%$ & 6006 & $65,4 \%$ & 5615 & $75,1 \%$ & 12888 & $52,6 \%$ \\
\hline Tercer escalón & 169 & $5,7 \%$ & 1050 & $11,4 \%$ & 1016 & $13,6 \%$ & 2235 & $9,5 \%$ \\
\hline
\end{tabular}

\begin{tabular}{|l|c|c|c|c|c|c|c|c|}
\hline & \multicolumn{7}{|c|}{ Grupo de edad (años) - Dorsalgia } & \\
\hline
\end{tabular}

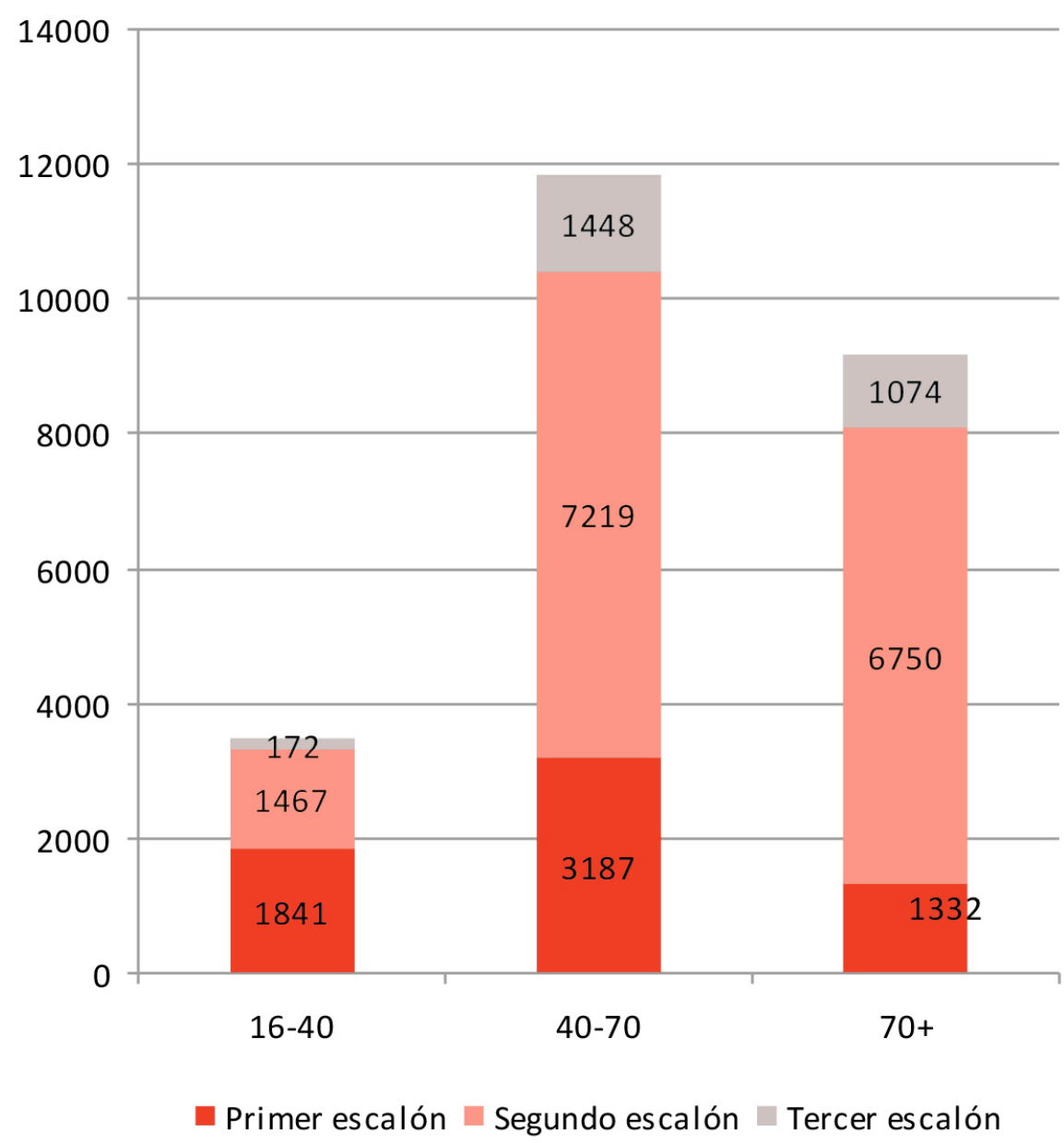

Gráfico 1. Distribución del tipo de analgesia en función del grupo mayor de edad. 
fueron tratados con el tercer escalón analgésico no presentaron un evento de fin de seguimiento. A los 14 días, las mismas proporciones fueron $74.5 \%$, $85 \%$ y $94 \%$, respectivamente. A los 30 días, las mismas proporciones fueron $67.4 \%, 78.6 \%$ y $91.7 \%$, respectivamente. En los tres puntos de corte, la tasa de supervivencia sin eventos de fin de seguimiento fue significativamente mayor en el grupo tratado con fármacos del tercer escalón analgésico $(P<0.0001)$. Por otra parte entre los pacientes con dorsalgia como motivo de consulta o diagnóstico, a los 7 días de haberse realizado la valoración en urgencias, $97.5 \%$ de los tratados con el primer escalón analgésico, $84.5 \%$ de los que fueron tratados con el segundo escalón analgésico y $97.3 \%$ de los que fueron tratados con el tercer escalón analgésico no presentaron un evento de fin de seguimiento. A los 14 días, las mismas proporciones fueron $73.6 \%, 79 \%$ y $95.6 \%$, respectivamente. A los 30 días, las mismas proporciones fueron $63.1 \%, 62.7 \%$ y $88.2 \%$, respectivamente. En los tres puntos de corte, la tasa de supervivencia sin eventos de fin de seguimiento fue significativamente mayor en el grupo tratado con fármacos del tercer escalón analgésico $(P<0.0001)$. Las curvas de supervivencia se exponen en el Gráfico 2.

La tabla 3 detalla el motivo por el cual se produjo la necesidad de reevaluación en función del periodo de tiempo en el que tuvo lugar dicha reevaluación. La proporción de pacientes que consultaron por un evento adverso antes del $7^{\circ}$ día y a los que se les había prescrito un medicamento del segundo escalón analgésico fue 8.2\%. Entre los 7-14 días dicha proporción fue $10.6 \%$ y entre los días $14-30$ fue $1.8 \%(P<0.05)$. En el caso de medicamentos del tercer escalón analgésico, las proporciones respectivas fueron $4.7 \%, 5.8 \%$ y $1.2 \%$, respectivamente $(P<0.05)$. El gráfico 3 expone las proporciones de pacientes que consultaron por eventos adversos en función del tiempo en que tuvo lugar dicha consulta.

\section{Discusión}

La frecuentación en los servicios de urgencia por pacientes que consultan por patología musculoesquelética se concentra fundamentalmente en procesos relacionados con el raquis[2]. El recidivismo en este tipo de patología implica una mayor demanda asistencial y consumo de recursos[4]. Entre los factores vinculados al recidivismo en urgencias por procesos relacionados con el raquis están el mal control analgésico, el bajo nivel cultural[4,5,13] y la demanda asistencial por eventos adversos derivados por la propia atención médica previa[14].

Nuestro estudio ha recogido una gran cantidad de registros de pacientes atendidos en urgencias por algias axiales clasificados en función del tipo de tratamiento pautado en los últimos 5 años. En términos generales el recidivismo detectado de forma global fue $18.02 \%$ al mes. Estudios previos sitúan esta proporción por encima de la estimada para el grueso de motivos de consulta por dolor mecánico no relacionado con traumatimos[2].

De acuerdo con nuestros resultados, la prescripción de fármacos del primer escalón analgésico es significativamente más prevalente que otros fármacos en el grupo de pacientes jóvenes y menos prevalente que otros fármacos en el grupo de pacientes mayores de 70 años. Tenemos dos hipótesis que justifican estos hallazgos: En primer lugar consideramos que los cambios degenerativos y el incremento de probabilidad de existencia de deformidades morfométricas en ancianos exigen una demanda de fármacos analgésicos más potentes que los incluidos en el primer analgésico. En el sentido inverso, la poca complejidad del dolor axial en pacientes jóvenes inclina al clínico a tratar a los pacientes con este tipo de fármacos.

El estudio de supervivencia demostró que tanto en casos de lumbalgia como de dorsalgia, la exposición de fármacos del tercer escalón analgésico supone una reducción significativa de la proporción 
Tasa de supervivencia - Lumbalgia

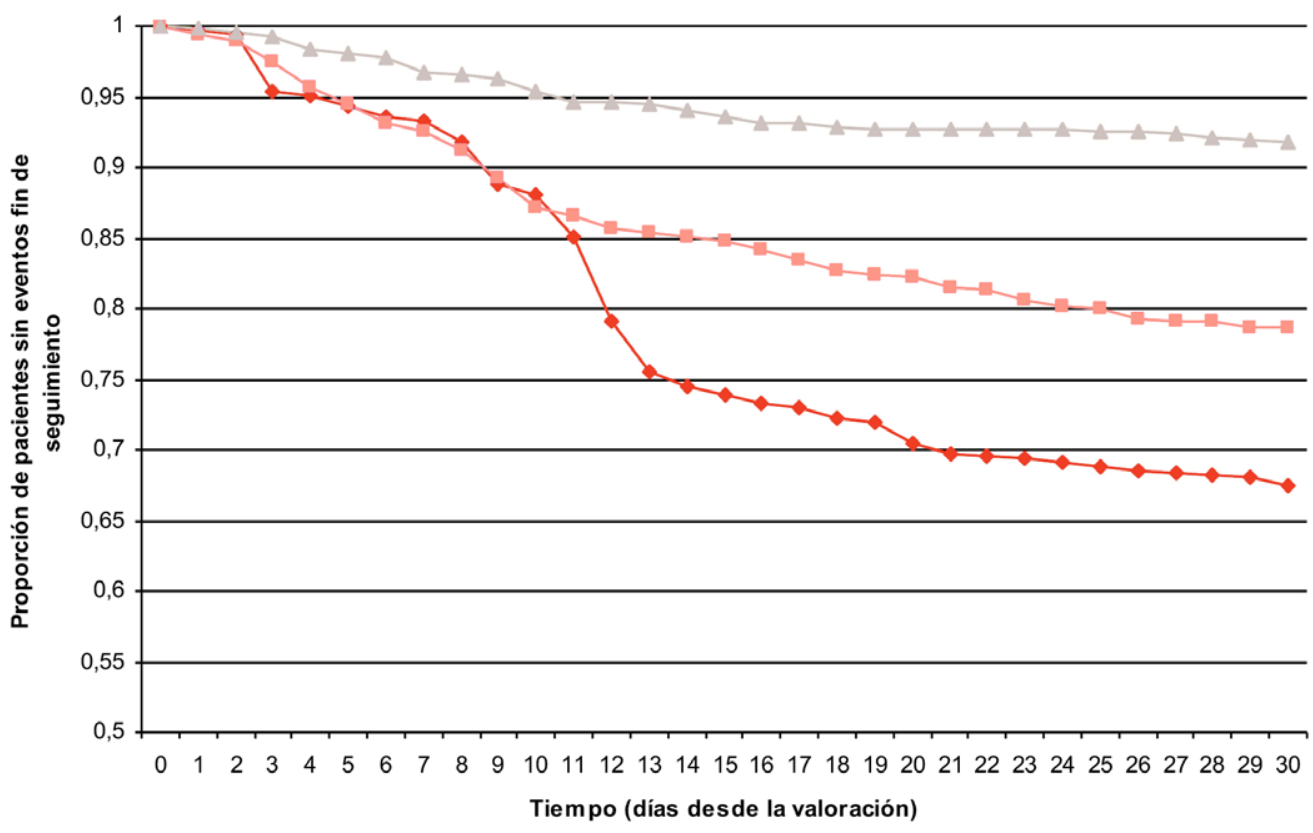

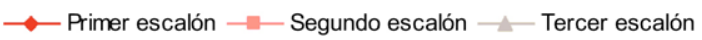

Tasa de supervivencia - Dorsalgia

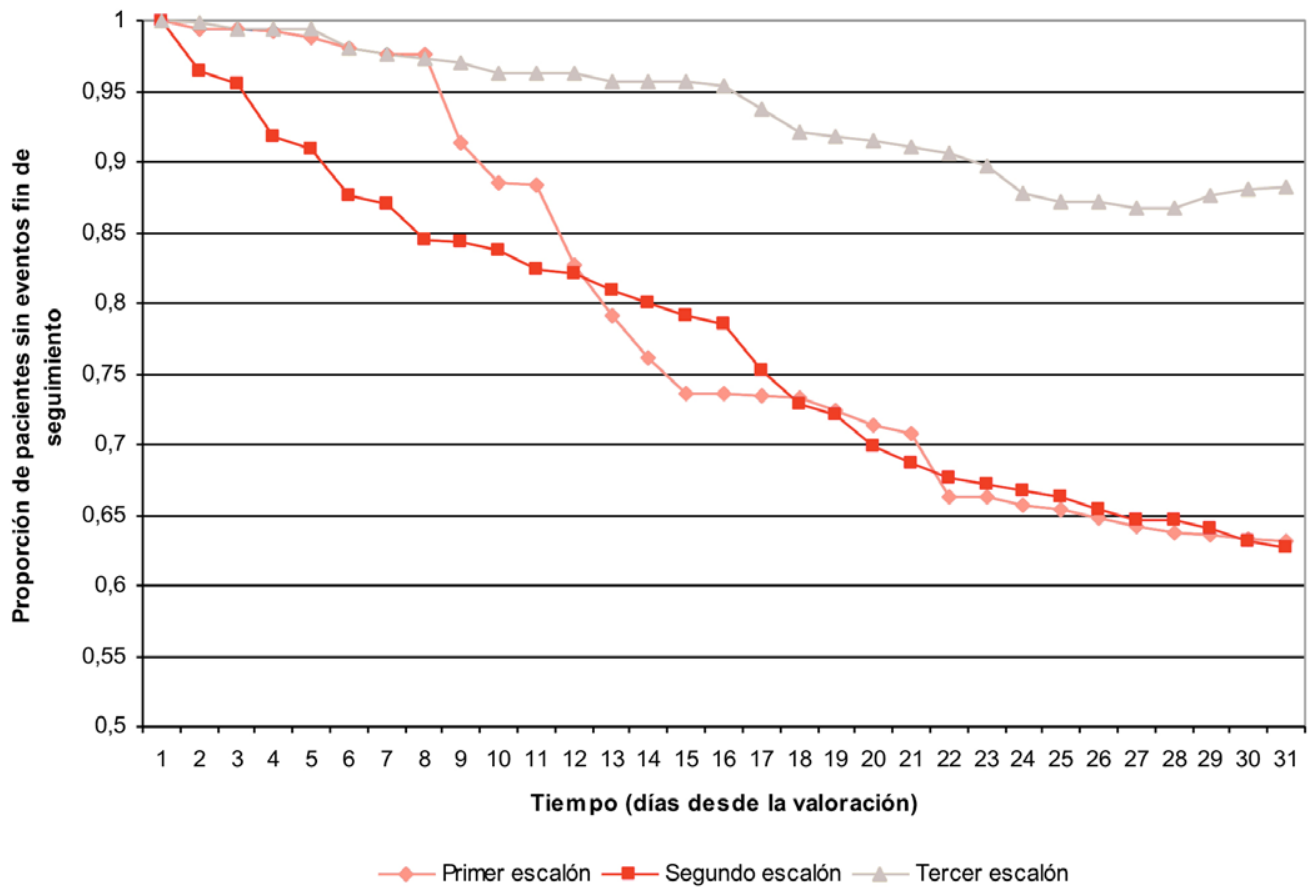

Gráfico 2. Curvas de supervivencia en función del grupo de tratamiento analgésico administrado. 
Tabla 3. Distribución de los motivos de nueva consulta de acuerdo al periodo en el que tuvo lugar y al grupo de tratamiento asignado. MCA: Mal control analgésico. EA: Efecto adverso. NC: No conocido/Ninguno de los previos.

\begin{tabular}{|c|c|c|c|c|c|c|c|c|c|c|c|c|}
\hline & \multicolumn{4}{|c|}{ Primer escalón } & \multicolumn{4}{|c|}{ Segundo escalón } & \multicolumn{4}{|c|}{ Tercer escalón } \\
\hline \multirow{3}{*}{$<7$ días } & \multirow{3}{*}{352} & MCA & 322 & $91,5 \%$ & \multirow{3}{*}{1361} & MCA & 1210 & $88,9 \%$ & \multirow{3}{*}{85} & MCA & 75 & $88,2 \%$ \\
\hline & & EA & 2 & $0,6 \%$ & & EA & 112 & $8,2 \%$ & & EA & 4 & $4,7 \%$ \\
\hline & & NC & 28 & $8,0 \%$ & & NC & 39 & $2,9 \%$ & & NC & 6 & $7,1 \%$ \\
\hline \multirow{3}{*}{ 7-14 días } & \multirow{3}{*}{1284} & MCA & 1267 & $98,7 \%$ & \multirow{3}{*}{1094} & MCA & 970 & $88,7 \%$ & \multirow{3}{*}{69} & MCA & 61 & $88,4 \%$ \\
\hline & & EA & 10 & $0,8 \%$ & & EA & 116 & $10,6 \%$ & & EA & 4 & $5,8 \%$ \\
\hline & & NC & 7 & $0,5 \%$ & & NC & 8 & $0,7 \%$ & & NC & 4 & $5,8 \%$ \\
\hline \multirow{3}{*}{ 14-30 días } & \multirow{3}{*}{513} & MCA & 502 & $97,9 \%$ & \multirow{3}{*}{1247} & MCA & 1130 & $90,6 \%$ & \multirow{3}{*}{85} & MCA & 79 & $92,9 \%$ \\
\hline & & EA & 2 & $0,4 \%$ & & EA & 23 & $1,8 \%$ & & EA & 1 & $1,2 \%$ \\
\hline & & NC & 9 & $1,8 \%$ & & NC & 94 & $7,5 \%$ & & NC & 5 & $5,9 \%$ \\
\hline
\end{tabular}

\section{Proporción de pacientes que consultaron por un evento adverso relacionado con el tratamiento antes del primer mes según grupo de tratamiento}

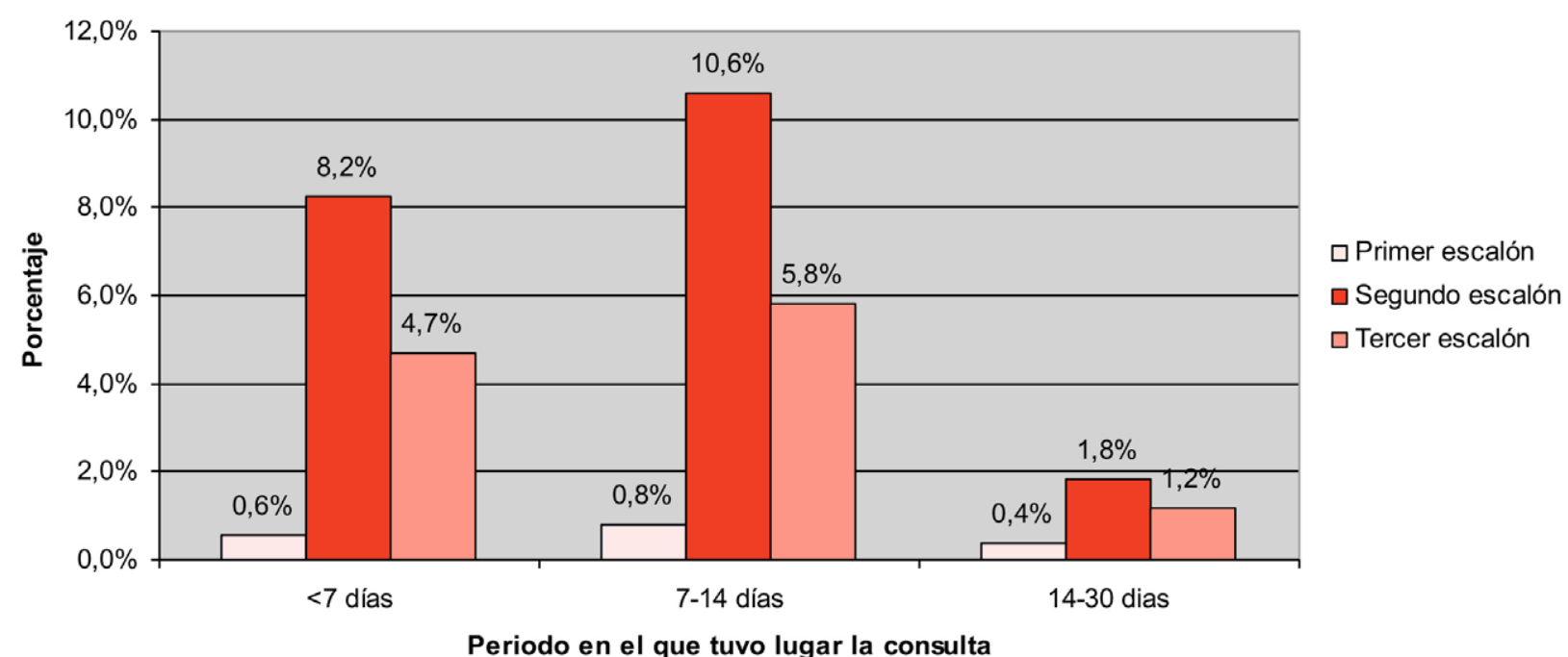

Gráfico 3. Proporción de eventos adversos como motivo de consulta repetida según el periodo de tiempo en que la consulta tuvo lugar y de acuerdo con el grupo de tratamiento administrado.

de pacientes sin necesidad de reconsulta que es evidente a partir del día 14 pero que en el caso de las lumbalgias se aprecia al $7^{\circ}$ día. Considerando la proporción de pacientes que reconsultó por eventos adversos atribuidos a la medicación encontramos que en todos los estratos de tiempo los motivados por fármacos del tercer escalón son menores que los motivados por fármacos del segundo escalón. 
Este estudio presenta las siguientes debilidades: Al tratarse de un estudio retrospectivo basado en registros clínicos, se ha identificado una proporción de pacientes en los que el motivo de reconsulta no ha sido reconocido y por lo tanto no ha sido clasificado. En algunos casos, esta proporción supera la de los eventos adversos por lo que es se reconocer que con toda probabilidad la proporción real de eventos adversos sea superior, aunque no más de la suma aritmética con los motivos no conocidos. La pérdida de seguimiento inherente a un estudio de supervivencia basado en seguimientos retrospectivos también es una debilidad. En nuestro medio, sin embargo, la consulta por dolor no suele diferirse por lo que es de esperar que los pacientes que pudieron perderse por causas no médicas finalmente consultaron fuera del ámbito de acceso de nuestros medios de recogida de información, en una proporción baja. Finalmente, hay que reconocer que en el diseño de este estudio no se contemplaron detalles clínicos como el proceso etiológico del dolor axial ni la administración concomitante de fármacos de uso profiláctico durante la pauta de opiaceos (antieméticos, laxantes, etc).

En virtud de los hallazgos expuestos y teniendo presentes las limitaciones de nuestro estudio, parece ser que la exposición terapéutica a fármacos del tercer escalón analgésico de la OMS reduce el recidivismo en patología del raquis sin incrementar significativamente los eventos adversos. Estudios más específicos y prospectivos basados en la práctica clínica habitual serían útiles para reafirmar nuestros resultados.

\section{Bibliografía}

1. Fialho SC de MS, de Castro GRW, Zimmermann AF, et al. Musculoskeletal system assessment in an emergency room. Rev Bras Reumatol 2011;51:240-8.

2. Guillén Astete C, Kaumi L, Tejada Sorados RM, et al. Prevalencia de la afección musculoesquelética no traumática como motivo de consulta y su impacto asistencial en un servicio de urgencias. SEMERGEN - Med Fam Published Online First: 2015. doi:10.1016/j.semerg.2015.02.003

3. Nassonov EL. Musculoskeletal disorders in Russia at the end of the 20th century. J Rheumatol Suppl 2003;67:56-8.

4. Ross MA, Hemphill RR, Abramson J, et al. The recidivism characteristics of an emergency department observation unit. Ann Emerg Med 2010;56:34-41. doi:10.1016/j. annemergmed.2010.02.012

5. Southerland LT, Richardson DS, Caterino JM, et al. Emergency department recidivism in adults older than 65 years treated for fractures. Am J Emerg Med 2014;32:1089-92. doi:10.1016/j. ajem.2014.05.005

6. Strand JJ, Bundrick JB. Clinical pearls in palliative medicine. Dis--Mon DM 2015;61:346-55. doi:10.1016/j. disamonth.2015.04.005

7. Grey C, Hall PB. Considerations of prescription opioid abuse and misuse among older adults in West Virginia--An UnderRecognized Population at Risk. W V Med J 2016;112:42-7.

8. Veal FC, Peterson GM. Pain in the Frail or Elderly Patient: Does Tapentadol Have a Role? Drugs Aging 2015;32:419-26. doi:10.1007/s40266-015-0268-7

9. Malec M, Shega JW. Pain management in the elderly. Med Clin North Am 2015;99:337-50. doi:10.1016/j.mcna.2014.11.007

10. Guillen Astete C, Botello Corzo D, Sobrino Grande C. Manejo analgésico de los aplastamientos osteoporóticos en urgencias. Estudio descriptivo sobre actitudes terapéuticas. Reumatol Clínica 2013;9 (Espec. Cong):121-2.

11. Guillen Astete $C$, Boteanu A, Luque Alarcón M, et al. Demanda asistencial urgente en pacientes con fracturas vertebrales en función de la administración de buprenorfina transdérmica u otros tratamientos analgésicos. 
12. WHO | WHO's pain ladder for adults. http://www.who.int/ cancer/palliative/painladder/en/ (accessed 7 Jul2013).

13. McNaughton CD, Collins SP, Kripalani S, et al. Low numeracy is associated with increased odds of 30-day emergency department or hospital recidivism for patients with acute heart failure. Circ Heart Fail 2013;6:40-6. doi:10.1161/ CIRCHEARTFAILURE.112.969477

14. Calder L, Pozgay A, Riff S, et al. Adverse events in patients with return emergency department visits. BMJ Qual Saf 2015;24:1428. doi:10.1136/bmjqs-2014-003194

\section{Opina sobre este artículo:}
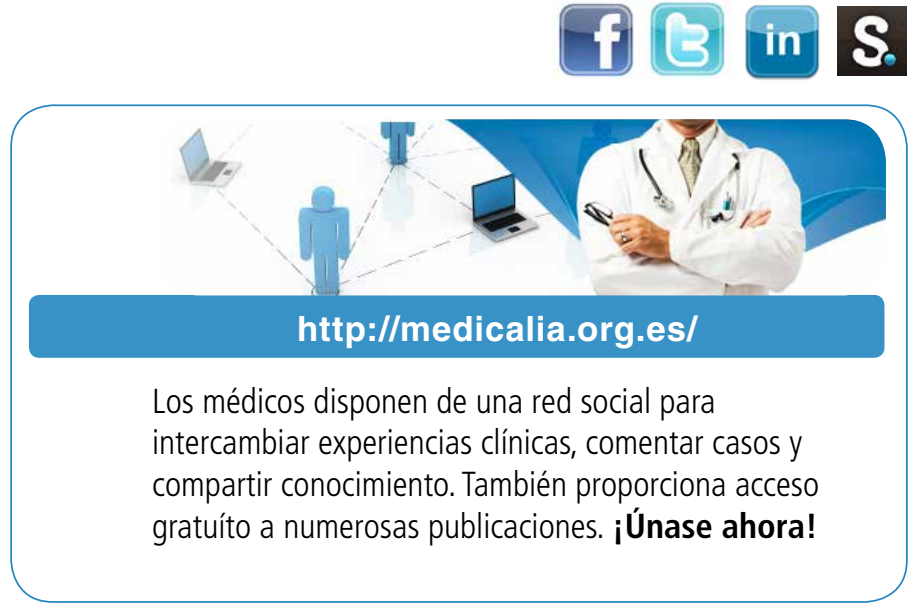

\section{Publish with iMedPub}

http://www.imed.pub

Acta Reumatológica es una revista que tiene por fin la difusión de estudios clínicos relacionados con aspectos prácticos del diagnóstico, tratamiento y seguimiento de pacientes con patología reumatológica, de estudios epidemiológicos relacionados con patología inflamatoria y musculoesquelética de presentación común o infrecuente en la práctica clínica tanto en población adulta como pediátrica, de casos clínicos de patología poco habitual o de presentaciones inhabituales de patología frecuente, de imágenes didácticas e ilustrativas en reumatología y del estado actual e innovación en la formación especializada en reumatología. 